\title{
A clinical study of hair manifestations in cancer patients on chemotherapy
}

\author{
Faizy Azeez ${ }^{1}$, Malcolm Pinto ${ }^{2, *}$, Manjunath Shenoy $\mathbf{M}^{3}$ \\ ${ }^{1}$ Post Graduate, ${ }^{2}$ Assistant Professor, ${ }^{3}$ Professor and HOD, Dept. of Dermatology, Yenepoya Medical College, Yenepoya \\ (Deemed to be University) Mangalore, Karnataka, India
}

*Corresponding Author:

Email: malcolmpinto@gmail.com

\begin{abstract}
Introduction: There has been an increase in cancer related morbidities; a significant part of it being related to therapies. Chemotherapy targets rapidly growing cells like skin, hair follicles and nail matrix. Hence a significant number of adverse effects are related to the skin and its appendages. Several factors may contribute to the severity of hair loss including the drug, dosage, schedule as well as hair care practices.

Aim: To determine the frequency and significance of hair manifestations in patients on cancer chemotherapy.

Materials and Methods: A total of 50 patients attending the oncology outpatient department or those admitted in the oncology ward Yenepoya Medical College Hospital, Mangalore between June 2017 and August 2017 were studied. All hair diseases, frequency and type of hair loss among patients were documented. Findings were tabulated and correlated with the cancer and the chemotherapy.

Results: Hair changes were mainly in the form of diffuse alopecia in $68 \%$, anagen effluvium was seen in $52 \%$ patients.

Conclusion: Though the adverse effects on hair are not serious, it can cause considerable anxiety to the patient. Hence prior counselling regarding the same needs to be advocated to improve the quality of life of the patient.
\end{abstract}

Keywords: Chemotherapy, Anagen Effluvium, Quality of life.

\section{Introduction}

Chemotherapy is an essential component in the multidisciplinary management of most cancers. Latest data reveals that the 5-year survival of all cancers has increased from $50 \%$ to $66 \%$ over the past 20 years. ${ }^{1}$ In the past decade there has been a tremendous increase in the number of newer chemotherapeutic drugs being introduced for cancer therapy. ${ }^{2}$ This has led to a better prognosis and a longer survival of patients affected by cancer. There has been an increase in the cancer related morbidities; a significant part of it being related to the therapies. Chemotherapy targets rapidly growing cells like skin, hair follicles and nail matrix. Hence a significant number of adverse effects are related to the skin and its appendages. ${ }^{3}$ The physical and psychological effects of cutaneous adverse events can significantly affect the Quality of life (QOL) of the patient resulting in a profound effect on the physical, emotional, financial and psychosocial health of patients. Patients may experience worry, frustration, depression and withdrawal from society because of these distressing side effects. ${ }^{4}$ The various factors that may contribute to the severity of hair loss including the classification of drug, dosage schedule as well as hair care practices. The present study has been undertaken to determine the frequency and significance of hair manifestations in patients on anticancer chemotherapy.

\section{Materials and Methods}

Fifty patients on chemotherapy referred from the department of oncology over a period of 3 months were included in our epidemiological study after obtaining ethical clearance. All cancer patients on chemotherapy after the $2^{\text {nd }}$ cycle were included. Patients on concomitant radiotherapy were excluded. Demography, type of malignancy and details of the therapy were recorded. All hair diseases, frequency and type of hair loss among patients were documented. Findings were tabulated and correlated with the cancer and the chemotherapy. A questionnaire on quality of life during chemotherapy treatment was given to each patient and the answers were evaluated.

\section{Results}

Out of the 50 cancer patients studied, 15 patients were on a single chemotherapy drug and 35 were on combined chemotherapy. The age range of patients in our study was 25-75 years with most of the patients $(56 \%)$ was in age group 40-60. Male preponderance was seen with a male to female ratio of 1.5:1. The various drugs used in the study were: paclitaxel, vincristine, vinblastine, 6-mercaptopurine, 5fluorouracil, cytarabine, gemcitabine, cisplatin, carboplatin, cyclophosphamide, doxorubicin, daunorubicin, epirubicin, hydroxyurea, CHOP (Cyclophosphamide, Doxorubicin, Vincristine, Prednisolone), and ABVD (Doxorubicin, Bleomycin, Vinblastine, Dacarbazine) regimens. Lung cancer was the most common cancer seen in 12 patients followed by breast cancer in 8 patients, acute lymphocytic leukaemia in 7 and cancer of colon in 6 patients. Details of the frequencies of the malignancies in our study have been shown in Graph 1. 


\section{Graph 1: Malignancies among patients on chemotherapy}

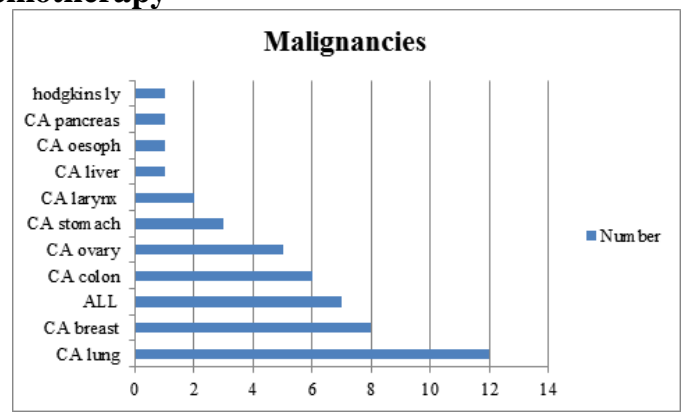

Table 1: Frequency of hair changes

\begin{tabular}{|l|c|}
\hline Hair changes & Number of patients \\
\hline Diffuse Alopecia & 34 \\
\hline Anagen effluvium & 26 \\
\hline Telogen effluvium & 6 \\
\hline
\end{tabular}

Table 2: Questionnaire on quality of life pertaining to hair changes

\begin{tabular}{|l|c|c|}
\hline QOL questionnaire & Yes & No \\
\hline $\begin{array}{l}\text { 1. If it will save my life, I will } \\
\text { undergo medical treatment } \\
\text { even if it makes my hair fall } \\
\text { out. }\end{array}$ & 40 & 0 \\
\hline $\begin{array}{l}\text { 2. Since it will all fall out } \\
\text { anyway, I bravely cut it off. }\end{array}$ & 28 & 12 \\
\hline $\begin{array}{l}\text { 3. I wear a hat or wig and feel } \\
\text { fashionable }\end{array}$ & 12 & 28 \\
\hline $\begin{array}{l}\text { 4. Wearing a wig, I worry } \\
\text { about people staring }\end{array}$ & 35 & 5 \\
\hline $\begin{array}{l}\text { 5. I don't want to see people } \\
\text { because I don't have hair. }\end{array}$ & 20 & 20 \\
\hline
\end{tabular}

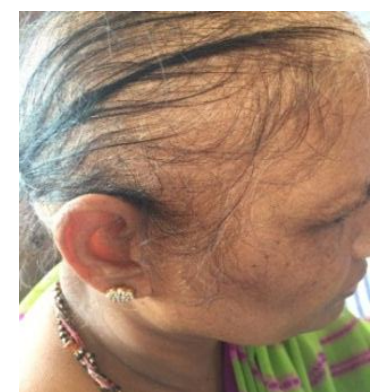

Fig. 1: Anagen effluvium secondary to 5Fluorouracil

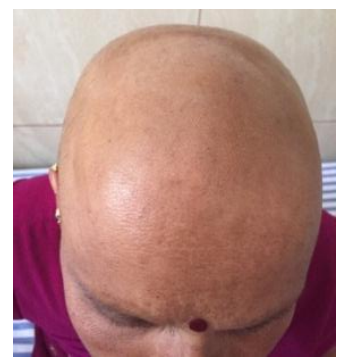

Fig. 2: Diffuse alopecia secondary to Cisplatin
Among the 50 patients studied, 40 patients showed hair changes, in the form of alopecia affecting the scalp. All patients had scalp involvement and 18 cases (36\%) had involvement of other sites. Out of the 40 cases 34 $(85 \%)$ presented with grade 2 and $6(15 \%)$ with grade 1 alopecia. In majority of the cases $(85 \%)$ the onset was within 4-5weeks of initiation of therapy. The hair changes observed have been tabulated in Table-1 Anagen effluvium was seen in $37(74 \%)$ patients and telogen effluvium in $6(12 \%)$ patients (Fig. 1, 2)

The components of the questionnaire on quality of life have been summarised in Table 2. All patients were willing to take chemotherapy despite the adverse effect of hair loss. Twenty-eight patients (70\%) were willing to cut the hair short, $12(30 \%)$ wanted to wear wigs, $35(85 \%)$ of the patients were worried about people staring if they wear wigs and 20 patients $(50 \%)$ did not want to meet people because of their hair loss.

\section{Discussion}

Chemotherapy forms an integral part in the multifaceted approach to the management of cancers. ${ }^{5}$ Chemotherapy agents having specific targets in the pathogenesis of malignancies may be toxic to the rapidly proliferating cells of the skin, hair and nails at therapeutic doses resulting in a wide array of cutaneous adverse events. ${ }^{6}$

In our study, carcinoma affecting the lung (24\%) and hematologic system (14\%) were the most common cancers among men. Among females the most common cancer was breast cancer $(16 \%)$ and carcinoma ovary $(10 \%)$. Kirthi et al reported that breast carcinoma (40\%), carcinoma cervix (11.4\%) and carcinoma ovary (8.6\%) were the most commonly encountered cancers in women and carcinoma of the lung $(10 \%)$ and urinary bladder $(8.3 \%)$ were the more frequently observed among men. ${ }^{7}$ In this study the most commonly prescribed chemotherapeutic drugs were cisplatin (50\%) followed by cyclophosphamide (20\%), carboplatin (12\%), paclitaxel (10\%) and 5 fluorouracil $(8 \%)$. In the study by Kirthi et al the drugs employed for chemotherapy were similar to those in this study with cisplatin being the most commonly prescribed followed by Adriamycin, 5- fluorouracil and cyclophosphamide. ${ }^{7}$ Alopecia is one of the most psychologically distressing adverse effects of cancer chemotherapy along with vomiting and nausea. In our study alopecia was seen in patients most commonly after 1-2 months of chemotherapy, which was also observed in a study by Chadha et al. ${ }^{8}$ Sixty-four percentage of patients rated alopecia secondary to chemotherapy as the most traumatic side effect and 9\% of patients considered not undergoing chemotherapy because of fear of hair loss. ${ }^{4}$ The observations made by Kirthi $\mathrm{C}$ et al and Pavey RA et al showed 37.8\% \& 37\% respectively. ${ }^{7,9}$ It is the most commonly reported cutaneous adverse event with chemotherapy. ${ }^{6}$ The commonly observed form of hair loss associated with 
chemotherapy is anagen effluvium. Anagen effluvium was the most common finding with 37 (74\%) of patients. This finding was in concordance with the study done by Pavey RA et al. ${ }^{9}$ In our study as no patients were treated with epidermal growth factor receptor (EGFR) inhibitors, hair changes such as trichomegaly and hair texture changes generally observed secondary to treatment with them were not observed. ${ }^{10-12}$

\section{Conclusion}

Accurate diagnosis and early recognition of hair manifestations of cancer chemotherapy may reduce significant morbidity, cosmetic disfigurement, and psychological distress. Educating patients about the potential side effects prior to initialization of therapy usually results in better acceptance and compliance by patients. Estimating the incidence and severity of adverse events as these may help to develop better intervention in terms of modifying doses or the drug regimen to minimize toxic effects. Non-correlation of type and severity of hair loss with dermoscopy and histopathology, small sample size and the duration of the study were the major limitations of our study. Hence there is a need for similar studies with larger sample size, comprehensive outlook of mucocutaneous adverse reactions and regular follow up to know the outcome of these manifestations which will enable validating the findings of this study.

\section{References}

1. Parkin D. Global cancer statistics in the year 2000. The Lancet Oncology. 2001;2(9):533-43.

2. Fabbrocini G, Izzo R, Panariello L, Monfrecola G. Skin reactions secondary to anticancer agents. EMJ Dermatol 2013; 1:38-43.

3. Sanches Junior JA, Brandt HR, Moure ER, Pereira GL, Criado PR. Adverse mucocutaneous reactions to chemotherapeutic agents - part I. An Bras Dermatol 2010;85(4):425-37.

4. Joshi S, Ortiz S, Witherspoon J, Rademaker A, West D, Anderson R et al. Effects of epidermal growth factor receptor inhibitor-induced dermatologic toxicities on quality of life. Cancer.2010; 116(16):3916-23.

5. Hasnat A, Poddar S, Sultana R, Sultana R, Akbor M, Azad M. Pattern of Adverse Drug Reactions Due to Cancer Chemotherapy in Tertiary Care Teaching Hospital in Bangladesh. Dhaka Univ J Pharm Sci. 2010;8(1).

6. Susser W, Whitaker-Worth D, Grant-Kels J. Mucocutaneous reactions to chemotherapy. J Am Acad Dermatol 1999;40(3):367-98.

7. Kirthi C, Afzal A, Reddy M, Ali SA, Yerramilli A, Sharma S. A study on the adverse effects of anticancer drugs in an oncology centre of a tertiary care hospital. Int J Pharm Pharm Sci 2014;6:580-3.

8. Chadha V, Shenoi SD. Hair loss in cancer chemotherapeutic patients. Indian J Dermatol Venereol Leprol. 2003;69(2):131-2.

9. Pavey RA, Kambil SM, Bhat RM. Dermatological adverse reactions to cancer chemotherapy. Indian J Dermatol Venereol Leprol 2015;81(4):434.

10. Lee M, Seo C, Kim S, Yang H, Lee H, Choi J et al. Cutaneous Side Effects in Non-Small Cell Lung Cancer
Patients Treated with Iressa (ZD1839), an Inhibitor of Epidermal Growth Factor. Acta Dermato-Venereologica. 2003;84(1):23-6.

11. Osio A, Mateus C, Soria J, Massard C, Malka D, Boige V et al. Cutaneous side-effects in patients on long-term treatment with epidermal growth factor receptor inhibitors. Br J Dermatol. 2009;161(3):515-521.

12. Busam K, Capodieci P, Motzer R, Kiehn T, Phelan D, Halpern A. Cutaneous side-effects in cancer patients treated with the ant epidermal growth factor receptor antibody C225. Br J Dermatol. 2001;144(6):1169-76. 